\title{
STABILITY OF MONTE CARLO $k$-EIGENVALUE SIMULATIONS WITH CMFD FEEDBACK
}

\author{
Kendra P. Keady ${ }^{\mathrm{a}}$, Edward W. Larsen ${ }^{\mathrm{b}, *}$ \\ ${ }^{a}$ CCS-2, Los Alamos National Laboratory \\ P.O. Box 1663 \\ Los Alamos, NM 87545 \\ ${ }^{b}$ University of Michigan Department of Nuclear Engineering and Radiological Sciences \\ 2355 Bonisteel Blvd. \\ Ann Arbor, MI 48109
}

\begin{abstract}
In this paper we perform a Fourier stability analysis of MC-CMFD, a hybrid Monte Carlo $k$-eigenvalue method that utilizes coarse mesh finite difference (CMFD) feedback. The MC-CMFD method is nonlinear and contains random statistical errors; both of these features are inconsistent with the direct application of a Fourier stability analysis. To accomplish this analysis, we first formulate a non-random iteration method that approximates MC-CMFD, by assuming an infinite number of Monte Carlo particles per cycle. Then we (i) linearize this method, and (ii) Fourier-analyze the linearized method to theoretically predict its convergence properties. Finally, we demonstrate by direct numerical simulations that the Fourier analysis of the linearized non-random method accurately predicts the stability and fission source convergence rate (during the inactive cycles) of the original nonlinear MC-CMFD method. We do this by comparing the predictions of the Fourier analysis to simulations that utilize (i) a high-fidelity $\mathrm{S}_{\mathrm{N}}$-CMFD code (which has no random statistical errors), and (ii) a MC-CMFD code (which has random statistical errors). The Fourier analysis and our two test codes confirm that the MC-CMFD method is stable if the optical thickness of the coarse grid (in the low-order CMFD calculation) is sufficiently small. However, the spectral radius increases monotonically with the coarse grid size, and if the latter exceeds a critical value, the MCCMFD method becomes unstable. We discuss some implications of our results for practical MC-CMFD simulations.
\end{abstract}

Keywords: Neutron Transport, Hybrid Methods, $k$-Eigenvalue Problems, Fourier Analysis

*Corresponding author: Email, edlarsen@umich.edu; Phone, +1 (734) 936-0124.

Preprint submitted to Journal of Computational Physics

April 13, 2016

(C) 2016. This manuscript version is made available under the Elsevier user license http://www.elsevier.com/open-access/userlicense/1.0/ 


\section{INTRODUCTION}

Typical Monte Carlo reactor core $k$-eigenvalue simulations are large and complex, requiring many "inactive cycles" to converge the fission source. In a 2009 publication, a hybrid Monte Carlo method was proposed in which the fission source convergence is "accelerated" at the end of each inactive Monte Carlo cycle using the results of a discrete low-order CMFD equation [1]. This method has since been implemented and tested in a handful of codes, with mixed results [2-6]. In the present paper, we use a Fourier analysis to study the stability of a non-random version of this method (obtained, in effect, by assuming an infinite number of Monte Carlo particles per cycle).

Considerable prior work has utilized Fourier analysis to study the convergence behavior of iterative methods for solving deterministic fixed-source problems $[7,8]$. This earlier work included the analysis of nonlinear methods, such as CMFD [8-10]. In such analyses, the original nonlinear method is first linearized, and then the linearized method is Fourier-analyzed. The Fourier analysis procedure involves (i) selecting a problem with a known solution, (ii) writing the initial solution guess as a set of periodic functions of differing frequency (often called "Fourier modes"), and (iii) determining the rate at which the slowestconverging function approaches (or diverges from) the true solution. In all cases discussed in the literature, the original nonlinear iterative methods are sufficiently robust that the linearized version of the method has nearly the same convergence properties as the original nonlinear method. Thus, predicting (via Fourier analysis) the stability and efficiency of the linearized method accurately predicts the stability and efficiency of the original nonlinear method.

In general, Fourier analysis can only be applied to simple problems with known analytical solutions. Thus, Fourier analysis cannot quantitatively predict the convergence rate of real-world simulations; rather, it is used to understand how the stability of a given iterative method changes as a function of the input parameters. This, in turn, provides valuable insight regarding the range of stable inputs for more realistic problems.

To date, Fourier analysis has only been used to study the stability of linear iterative schemes for deterministic problems. It is not known how to include statistical noise in a Fourier stability analysis. Thus, to accomplish the work outlined in this paper, we analyze a non-random version of the MC-CMFD method, obtained by (mathematically) assuming an infinite number of Monte Carlo particles per cycle. This "non-random MC-CMFD" method has a straightforward mathematical formulation, which can be linearized and then Fourier-analyzed.

To our knowledge, only one previous publication, by S.G. Hong, K.-S. Kim, and J.S. Song, has addressed the Fourier analysis of iterative methods for $k$ eigenvalue problems [11] - and that prior work considered only iterative methods for deterministic simulations. (The iteration processes for deterministic and hybrid Monte Carlo $k$-eigenvalue simulations are related, but different.) We

are particularly interested in the effect on stability of the optical thickness of a coarse mesh in MC-CMFD simulations [5]. The work in the present paper has little direct overlap with the previous work in [11]. 
We emphasize that our Fourier analysis is only valid for the hypothetical nonrandom MC-CMFD method having an infinite number of Monte Carlo particles per cycle. While this idealized method cannot be simulated in practice, the infinite-particle Fourier analysis predicts a "best-case" convergence rate for the real method with a finite number of particles. If the MC-CMFD method is unstable in the non-random infinite-particle limit, it cannot be expected to be stable for a practical simulation with a finite number of particles per cycle. In fact, we observe in our numerical experiments that if the non-random MCCMFD method has a spectral radius less than but sufficiently close to unity, then a "fixed" amount of random noise per cycle can actually drive the random MCCMFD method unstable. Therefore, again, the results of the Fourier analysis represent a "best-case" convergence rate.

Also, the Fourier analysis of the non-random MC-CMFD method only describes the convergence properties of the MC-CMFD method during inactive cycles, when the error in the fission source is greater than the random statistical error. During active cycles, when the error in the fission source is dominated by random statistical fluctuations, the Fourier analysis is not relevant.

Not surprisingly, we find that for non-random MC-CMFD, reducing the size of the coarse mesh reduces the spectral radius and improves the stability of the method. (The same is basically true for deterministic fixed-source CMFD problems [8].) We also find that if the coarse mesh exceeds a critical optical thickness, the non-random MC-CMFD method becomes unstable. (This is also true for fixed-source problems with sufficiently high scattering ratios [8].) Since the deterministic CMFD and MC-CMFD methods differ, their stability properties differ for a specified coarse mesh thickness. Nonetheless, for both methods (with a sufficiently high scattering ratio for deterministic CMFD), the following basic trends are the same: the spectral radius (i) is less than unity for small coarse mesh thicknesses, (ii) monotonically increases as the coarse mesh width increases, and (iii) exceeds unity when the coarse mesh width exceeds a critical value.

In the present paper we derive theoretical predictions for the spectral radius $\rho$ of the non-random MC-CMFD method as a function of the coarse mesh size. We include comparisons with monoenergetic and two-group numerical simulations, demonstrating that the theoretical predictions are accurate. Our numerical demonstrations employ (i) a finite-differenced $S_{N}$ code, especially written to simulate the non-random MC-CMFD method, and (ii) a hybrid MC-CMFD code using a large (but not infinite) number of Monte Carlo particles per cycle. Our results show that the non-random CMFD method is stable when the optical thickness of a coarse grid cell (in the low-order CMFD calculation) is small. However, the spectral radius of the non-random MC-CMFD method increases as the optical thickness of the coarse grid cells increases, to the point where the former exceeds unity when the latter exceeds a critical value. (At this point, the non-random MC-CMFD method becomes unstable.) In all cases, the Fourier analysis and the finite-differenced $\mathrm{S}_{\mathrm{N}}$ code produced nearly identical estimates of $\rho$.

The comparisons between the Fourier analysis and the MC-CMFD code were 
less close, due the the effects of statistical noise. However, for problems in which $0.5<\rho<0.98$, very good agreement was seen. (For problems with $\rho<0.5$, convergence was so rapid that with statistical noise, it was very difficult to obtain accurate Monte Carlo estimates of $\rho$. Similarly, with $\rho>0.98$, we found that a small amount of noise can drive an otherwise "barely stable" configuration unstable).

Overall, the Fourier analysis accurately predicts the rate of convergence (or divergence) of the fission source in MC-CMFD problems during inactive cycles, when the error in the fission source exceeds the random statistical errors. If the non-random MC-CMFD method is unstable for a particular set of parameters, then surely it would be unwise to employ this method using the same parameters with a finite number of particles per cycle. We are not aware of any previouslypublished work that addresses the issue of numerical instabilities in MC-CMFD simulations.

A lesson for more general computational methods can be gleaned from the work presented in this paper. It is well-known that iterative methods for deterministic methods can be unstable, due to inherent properties of the mathematical equations that describe the method. It is also well-known that Monte Carlo methods have statistical errors, which can usually be controlled by using a sufficiently large number of trials. This paper shows that hybrid methods - which combine elements of both Monte Carlo and deterministic methods - can suffer from both statistical errors and fundamental instabilities, and that instabilities can occur even if the number of Monte Carlo particles used is (hypothetically) infinite.

The remainder of this paper is organized as follows. In Section 2, the nonlinear non-random MC-CMFD iteration strategy for $k$-eigenvalue problems is described. In Section 3, this method is linearized, and in Section 4, the continuous linearized strategy is approximated by a discrete problem and Fourier-analyzed.

In Section 5, the the Fourier analysis of Section 4 is employed to predict the stability and convergence of the MC-CMFD method for both a monoenergetic and a two-group problem, and comparisons are made with independent deterministic and Monte Carlo test codes. Our results confirm the accuracy of the Fourier analysis predictions. The paper concludes in Section 6 with a discussion on the implications of this work for practical MC-CMFD simulations, and possible extensions of the results for more general problems.

Finally, the work presented in this paper was part of the Ph.D. thesis of the first author (K.P.K.).

\section{CMFD ITERATION STRATEGY FOR MONTE CARLO $k$-EIGENVALUE PROBLEMS}

Here we describe the non-random MC-CMFD iteration strategy for solving Monte Carlo $k$-eigenvalue problems. (This method is simply the MC-CMFD method [1], assuming an infinite number of particles per cycle.) We consider the following one-group, planar-geometry $k$-eigenvalue problem on the domain 
$0 \leq x \leq X$ with periodic boundary conditions:

$$
\begin{array}{r}
\mu \frac{d}{d x} \psi(x, \mu)+\Sigma_{t} \psi(x, \mu)-\frac{\Sigma_{s}}{2} \phi(x)=\frac{\nu \Sigma_{f}}{2 k} \phi(x), \\
0 \leq x \leq X,-1 \leq \mu \leq 1, \\
\psi(0, \mu)=\psi(X, \mu),-1 \leq \mu \leq 1,
\end{array}
$$

where

$$
\phi(x)=\int_{-1}^{1} \psi(x, \mu) d \mu .
$$

It is conceptually straightforward to generalize this system of equations (and the subsequent Fourier analysis) to an arbitrary number of energy groups. We present the monoenergetic case here for simplicity, but will consider both monoenergetic and two-group problems in Section 5.

The $l^{\text {th }}$ MC-CMFD iteration (or "cycle") begins with Eqs. (1), written with iteration superscripts as follows:

$$
\begin{array}{r}
\mu \frac{d}{d x} \psi^{(l+1 / 2)}(x, \mu)+\Sigma_{t} \psi^{(l+1 / 2)}(x, \mu)-\frac{\Sigma_{s}}{2} \phi^{(l+1 / 2)}(x)=\frac{\nu \Sigma_{f}}{2 k^{(l)}} \phi^{(l)}(x), \\
0 \leq x \leq X, \quad-1 \leq \mu \leq 1, \\
\psi^{(l+1 / 2)}(0, \mu)=\psi^{(l+1 / 2)}(X, \mu), \quad-1 \leq \mu \leq 1,
\end{array}
$$

where

$$
\phi^{(l+1 / 2)}(x)=\int_{-1}^{1} \psi^{(l+1 / 2)}(x, \mu) d \mu .
$$

In contrast to standard deterministic methods, the Monte Carlo transport simulation shown in Eq. (2a) treats scattering implicitly; the scattering source is treated as being fully converged, because Monte Carlo methods simulate full particle histories (from birth until death) within each cycle. (This is not to be confused with "implicit capture," a common Monte Carlo variance reduction technique.) Eqs. (2) are not discretized in space or angle, because Monte Carlo simulations involve no such discretizations. Finally, Eqs. (2) have no terms that describe random errors. These equations apply to Monte Carlo simulations only in the limit in which the number of particles per cycle is sufficiently large that statistical errors can be ignored.

To proceed, we impose a "coarse" spatial grid on the system, with $J$ cells of width $\Delta_{j}(1 \leq j \leq J)$. During the solution of Eqs. (2), the following quantities are tallied on the coarse grid for $1 \leq j \leq J$. (Capital letters $(\Phi, \Delta)$ denote coarse-grid quantities, while lower-case letters $(\psi, \phi)$ denote continuous quantities.)

$$
\Phi_{j}^{(l+1 / 2)}=\frac{1}{\Delta_{j}} \int_{x_{j-1 / 2}}^{x_{j+1 / 2}} \phi^{(l+1 / 2)}(x) d x
$$




$$
\begin{aligned}
\Phi_{1, j \pm 1 / 2}^{(l+1 / 2)} & =\int_{-1}^{1} \mu \psi^{(l+1 / 2)}\left(x_{j \pm 1 / 2}, \mu\right) d \mu \\
\Sigma_{a, j}^{(l+1 / 2)} & =\frac{\int_{x_{j-1 / 2}}^{x_{j+1}} \Sigma_{a}(x) \phi^{(l+1 / 2)}(x) d x}{\int_{x_{j-1 / 2}}^{x_{j+1 / 2}} \phi^{(l+1 / 2)}(x) d x}, \\
\Sigma_{t, j}^{(l+1 / 2)} & =\frac{\int_{x_{j-1 / 2}}^{x_{j+1 / 2} \Sigma_{t}(x) \phi^{(l+1 / 2)}(x) d x}}{\int_{x_{j-1 / 2}}^{x_{j+1 / 2}} \phi^{(l+1 / 2)}(x) d x}, \\
\nu \Sigma_{f, j}^{(l+1 / 2)} & =\frac{\int_{x_{j-1 / 2}}^{x_{j+1 / 2}} \nu \Sigma_{f}(x) \phi^{(l+1 / 2)}(x) d x}{\int_{x_{j-1 / 2}}^{x_{j+1 / 2}} \phi^{(l+1 / 2)}(x) d x} .
\end{aligned}
$$

Also, the following interior-edge quantities are calculated:

$$
\begin{aligned}
\tilde{D}_{j+1 / 2}^{(l+1 / 2)} & =\frac{2}{3}\left(\frac{1}{\Sigma_{t, j}^{(l+1 / 2)} \Delta_{j}+\Sigma_{t, j+1}^{(l+1 / 2)} \Delta_{j+1}}\right) \\
\hat{D}_{j+1 / 2}^{(l+1 / 2)} & =\frac{\Phi_{1, j+1 / 2}^{(l+1 / 2)}+\tilde{D}_{j+1 / 2}^{(l+1 / 2)}\left(\Phi_{j+1}^{(l+1 / 2)}-\Phi_{j}^{(l+1 / 2)}\right)}{\Phi_{j+1}^{(l+1 / 2)}+\Phi_{j}^{(l+1 / 2)}}
\end{aligned}
$$

and the exterior edge quantities are calculated using the periodic boundary condition:

$$
\begin{aligned}
\tilde{D}_{1 / 2}^{(l+1 / 2)} & =\tilde{D}_{J+1 / 2}^{(l+1 / 2)} \\
& =\frac{2}{3}\left(\frac{1}{\Sigma_{t, 1}^{(l+1 / 2)} \Delta_{1}+\Sigma_{t, J}^{(l+1 / 2)} \Delta_{J}}\right), \\
\hat{D}_{1 / 2}^{(l+1 / 2)} & =\hat{D}_{J+1 / 2}^{(l+1 / 2)} \\
& =\frac{\Phi_{1,1 / 2}^{(l+1 / 2)}+\tilde{D}_{1 / 2}^{(l+1 / 2)}\left(\Phi_{1}^{(l+1 / 2)}-\Phi_{J}^{(l+1 / 2)}\right)}{\Phi_{1}^{(l+1 / 2)}+\Phi_{J}^{(l+1 / 2)}} .
\end{aligned}
$$

In practice, the coarse-grid cross-sections and other quantities are computed using one of two MC tally types. Collision estimators are only accumulated when a particle undergoes a collision with the material. The expression for a collision estimate of some coarse-grid cross-section in cell $j\left(\Sigma_{X, j}\right)$ is written

$$
\Sigma_{X, j}=\frac{\sum_{c=1}^{C_{j}} \Sigma_{X}\left(x_{c}\right) \frac{w_{c}}{\Sigma_{t}\left(x_{c}\right)}}{\sum_{c=1}^{C_{j}} \frac{w_{c}}{\Sigma_{t}\left(x_{c}\right)}},
$$

where $C_{j}$ is the total number of collisions in cell $j, \Sigma_{t}\left(x_{c}\right)$ is the total cross section at the collision point, and $w_{s}$ is the weight of the colliding particle.

Track-length estimators are accumulated both when a particle undergoes a collision and when it crosses a cell boundary. The track-length estimate of $\Sigma_{X, j}$ can be expressed using

$$
\Sigma_{X, j}=\frac{\sum_{s=1}^{S_{j}} \Sigma_{X}\left(x_{s}\right) l_{s} w_{s}}{\sum_{s=1}^{S_{j}} l_{s} w_{s}},
$$


where $S_{j}$ is the number of path-length segments in cell $j, l_{s}$ is the length of segment $s$, and $w_{s}$ is the weight of the particle along segment $s$. The tracklength estimator takes into account particles that stream through a spatial cell without colliding, while the collision estimator does not. For this reason, a track-length tally of some quantity will typically contain less statistical noise than a collision tally of the same quantity (for a fixed number of particles).

At the end of the Monte Carlo cycle, tallied estimates of $\Sigma_{a, j}^{(l+1 / 2)}, \nu \Sigma_{f, j}^{(l+1 / 2)}$, $\hat{D}_{j+1 / 2}^{(l+1 / 2)}$, and $\tilde{D}_{j+1 / 2}^{(l+1 / 2)}$ are used to build the coefficients of the CMFD system. The low-order discrete CMFD equations take the form:

$$
\begin{gathered}
\Phi_{1, j+1 / 2}^{(l+1)}-\Phi_{1, j-1 / 2}^{(l+1)}+\Sigma_{a, j}^{(l+1 / 2)} \Phi_{j}^{(l+1)} \Delta_{j}=\frac{\nu \Sigma_{f, j}^{(l+1 / 2)}}{k^{(l+1)}} \Phi_{j}^{(l+1)} \Delta_{j}, \quad 1 \leq j \leq J \\
\Phi_{1, j+1 / 2}^{(l+1)}=\tilde{D}_{j+1 / 2}^{(l+1 / 2)}\left(\Phi_{j+1}^{(l+1)}-\Phi_{j}^{(l+1)}\right)+\hat{D}_{j+1 / 2}^{(l+1 / 2)}\left(\Phi_{j+1}^{(l+1)}+\Phi_{j}^{(l+1)}\right) \\
\Phi_{1,1 / 2}^{(l+1)}=\Phi_{1, J+1 / 2}^{(l+1)} \\
1=\frac{1}{J} \sum_{j=1}^{J} \Phi_{j}^{(l+1)} .
\end{gathered}
$$

On convergence, Eq. (6a) is simply the neutron balance equation, obtained by integrating Eq. (1a) over angle and the $j^{t h}$ coarse cell. Also on convergence, Eq. (6b) reduces to the identity $\Phi_{1, j+1 / 2}=\Phi_{1, j+1 / 2}$. (The motivation behind the specific construction of Eq. (6b) is Fick's Law.) Finally, on convergence Eq. (6c) reduces to the condition from Eq. (1b) that the converged scalar flux must be periodic. For these reasons, the low-order discrete Eqs. (6) are fully consistent with the high-order continuous Eqs. (1), and on convergence, the solution of these equations would be the true scalar fluxes, volume-averaged over each coarse cell.

To proceed, the "current" terms $\Phi_{1, j+1 / 2}^{(l+1)}$ in Eqs. (6a) and (6b) are eliminated to form an algebraic system involving only the coarse-grid scalar fluxes, $\Phi_{j}^{(l+1)}$ (this system is not tridiagonal because of the periodic boundary condition). The solution of Eqs. (6) yields a new estimate of the $k$-eigenvalue and coarse-grid scalar flux, the latter of which is used to scale the continuous fine-grid fission source for the next cycle:

$$
\phi^{(l+1)}(x)=\phi^{(l+1 / 2)}(x)\left[\frac{\Phi_{j}^{(l+1)}}{\Phi_{j}^{(l+1 / 2)}}\right], \quad x_{j-1 / 2} \leq x \leq x_{j+1 / 2}, \quad 1 \leq j \leq J .
$$

This completes the description of the MC-CMFD iteration strategy [1]. To summarize, a single iteration "cycle" has the following steps: (i) perform a Monte 
Carlo calculation, using the previous-cycle estimate of the fission source term (or initial guess if $l=0$ ) [Eqs. (2)], (ii) use Monte Carlo tallies to form the coefficients of the CMFD system [Eqs. (3)], (iii) solve the CMFD system [Eqs. (6)] to obtain an updated estimate of the coarse-grid scalar flux and eigenvalue, and (iv) scale the fine-grid fission source using the updated coarse-grid scalar flux [Eq. (7)]. If the number of Monte Carlo particles is allowed to limit to $\infty$, then the preceding equations mathematically define the "non-random MCCMFD" method, which we will next linearize, and then Fourier-analyze.

\section{LINEARIZED NON-RANDOM MC-CMFD METHOD}

To linearize the non-random MC-CMFD $k$-eigenvalue iteration method defined above, we consider a special problem in which the exact eigenfunction is constant (independent of space and angle). Specifically, we consider a spatially uniform system with periodic boundary conditions:

$$
\begin{array}{r}
\mu \frac{d}{d x} \psi^{(l+1 / 2)}(x, \mu)+\Sigma_{t} \psi^{(l+1 / 2)}(x, \mu)-\frac{\Sigma_{s}}{2} \phi^{(l+1 / 2)}(x)=\frac{\nu \Sigma_{f}}{2 k^{(l)}} \phi^{(l)}(x), \\
0 \leq x \leq X,-1 \leq \mu \leq 1, \\
\psi^{(l+1 / 2)}(0, \mu)=\psi^{(l+1 / 2)}(X, \mu), \quad-1 \leq \mu \leq 1 .
\end{array}
$$

The CMFD grid is assumed to be uniform, with $J$ cells of width $\Delta$. The coarsegrid cross sections and diffusivities $(\tilde{D})$ are known exactly:

$$
\begin{aligned}
\Sigma_{a, j}^{(l+1 / 2)} & =\Sigma_{a}, \\
\Sigma_{t, j}^{(l+1 / 2)} & =\Sigma_{t}, \\
\nu \Sigma_{f, j}^{(l+1 / 2)} & =\nu \Sigma_{f}, \\
\tilde{D}_{j+1 / 2}^{(l+1 / 2)} & =\tilde{D}=\frac{1}{3 \Sigma_{t} \Delta},
\end{aligned}
$$

while the remainder of the equations become:

$$
\begin{aligned}
& \phi^{(l+1 / 2)}(x)=\int_{-1}^{1} \psi^{(l+1 / 2)}(x, \mu) d \mu \\
& \Phi_{j}^{(l+1 / 2)}=\frac{1}{\Delta} \int_{x_{j-1 / 2}}^{x_{j+1 / 2}} \int_{-1}^{1} \psi^{(l+1 / 2)}(x, \mu) d \mu d x \\
& \Phi_{1, j+1 / 2}^{(l+1 / 2)}=\int_{-1}^{1} \mu \psi^{(l+1 / 2)}\left(x_{j+1 / 2}, \mu\right) d \mu \\
& \hat{D}_{j+1 / 2}^{(l+1 / 2)}=\frac{\Phi_{1, j+1 / 2}^{(l+1 / 2)}+\tilde{D}\left(\Phi_{j+1}^{(l+1 / 2)}-\Phi_{j}^{(l+1 / 2)}\right)}{\Phi_{j+1}^{(l+1 / 2)}+\Phi_{j}^{(l+1 / 2)}} \\
& \Phi_{1, j+1 / 2}^{(l+1)}-\Phi_{1, j-1 / 2}^{(l+1)}+\Sigma_{a} \Phi_{j}^{(l+1)} \Delta=\frac{\nu \Sigma_{f}}{k^{(l+1)}} \Phi_{j}^{(l+1)} \Delta, \quad 1 \leq j \leq J,
\end{aligned}
$$




$$
\begin{gathered}
\Phi_{1, j+1 / 2}^{(l+1)}=\tilde{D}\left(\Phi_{j+1}^{(l+1)}-\Phi_{j}^{(l+1)}\right)+\hat{D}_{j+1 / 2}^{(l+1 / 2)}\left(\Phi_{j+1}^{(l+1)}+\Phi_{j}^{(l+1)}\right), \\
\Phi_{1,1 / 2}^{(l+1 / 2)}=\Phi_{1, J+1 / 2}^{(l+1 / 2)} \\
1=\frac{1}{J} \sum_{j=1}^{J} \Phi_{j}^{(l+1)} .
\end{gathered}
$$

On convergence, this problem has the following exact solution:

$$
\begin{aligned}
\psi(x, \mu) & =\frac{1}{2}, \\
\Phi_{j}=\phi(x) & =1, \\
\Phi_{1, j+1 / 2} & =0, \\
k & =\frac{\nu \Sigma_{f}}{\Sigma_{a}} .
\end{aligned}
$$

To proceed, we define the following linear expansions around the exact solution (with $\epsilon \ll 1$ ):

$$
\begin{aligned}
\psi^{(l+1 / 2)}(x, \mu) & =\frac{1}{2}+\epsilon \tilde{\psi}^{(l+1 / 2)}(x, \mu), \\
\phi^{(l+1 / 2)}(x) & =1+\epsilon \tilde{\phi}^{(l+1 / 2)}(x), \\
\phi^{(l+1)}(x) & =1+\epsilon \tilde{\phi}^{(l+1)}(x) \\
\Phi_{j}^{(l+1 / 2)} & =1+\epsilon \tilde{\Phi}_{j}^{(l+1 / 2)} \\
\Phi_{j}^{(l+1)} & =1+\epsilon \tilde{\Phi}_{j}^{(l+1)} \\
\Phi_{1, j+1 / 2}^{(l+1 / 2)} & =0+\epsilon \tilde{\Phi}_{1, j+1 / 2}^{(l+1 / 2)} \\
\Phi_{1, j+1 / 2}^{(l+1)} & =0+\epsilon \tilde{\Phi}_{1, j+1 / 2}^{(l+1)} \\
\hat{D}_{j+1 / 2}^{(l+1 / 2)} & =0+\epsilon \hat{d}_{j+1 / 2}^{(l+1 / 2)} \\
\frac{1}{k^{(l+1)}} & =\frac{\Sigma_{a}}{\nu \Sigma_{f}}+\epsilon \delta^{(l+1)}
\end{aligned}
$$

Since the coarse-grid cross sections and diffusion coefficients are known exactly, these quantities are not expanded. Inserting Eqs. (12) into Eqs. (8) and (10), we find that the $O(1)$ terms are satisfied exactly, but the $O(\epsilon)$ terms are not. Equating the $O(\epsilon)$ terms, we obtain:

$$
\begin{gathered}
\mu \frac{d}{d x} \tilde{\psi}^{(l+1 / 2)}(x, \mu)+\Sigma_{t} \tilde{\psi}^{(l+1 / 2)}(x, \mu)-\frac{\Sigma_{s}}{2} \tilde{\phi}^{(l+1 / 2)}(x)=\frac{\nu \Sigma_{f}}{2} \delta^{(l)}+\frac{\Sigma_{a}}{2} \tilde{\phi}^{(l)}(x) \\
0 \leq x \leq X,-1 \leq \mu \leq 1, \\
\tilde{\psi}^{(l+1 / 2)}(0, \mu)=\tilde{\psi}^{(l+1 / 2)}(X, \mu),-1 \leq \mu \leq 1,
\end{gathered}
$$




$$
\begin{aligned}
& \tilde{\phi}^{(l+1 / 2)}(x)=\int_{-1}^{1} \tilde{\psi}^{(l+1 / 2)}(x, \mu) d \mu, \\
& \tilde{\Phi}_{j}^{(l+1 / 2)}=\frac{1}{\Delta} \int_{x_{j-1 / 2}}^{x_{j+1 / 2}} \int_{-1}^{1} \tilde{\psi}^{(l+1 / 2)}(x, \mu) d \mu d x, \\
& \tilde{\Phi}_{1, j+1 / 2}^{(l+1 / 2)}=\int_{-1}^{1} \mu \tilde{\psi}^{(l+1 / 2)}\left(x_{j+1 / 2}, \mu\right) d \mu, \\
& \hat{d}_{j+1 / 2}^{(l+1 / 2)}=\frac{1}{2}\left(\tilde{\Phi}_{1, j+1 / 2}^{(l+1 / 2)}+\tilde{D}\left(\tilde{\Phi}_{j+1}^{(l+1 / 2)}-\tilde{\Phi}_{j}^{(l+1 / 2)}\right)\right), \\
& \nu \Sigma_{f} \delta^{(l+1)} \Delta=\left(\tilde{\Phi}_{1, j+1 / 2}^{(l+1)}-\tilde{\Phi}_{1, j-1 / 2}^{(l+1)}\right) \\
& \tilde{\Phi}_{1, j+1 / 2}^{(l+1)}=-\tilde{D}\left(\tilde{\Phi}_{j+1}^{(l+1)}-\tilde{\Phi}_{j}^{(l+1)}\right)+2 \hat{d}_{j+1 / 2}^{(l+1 / 2)}, \\
& \tilde{\Phi}_{1,1 / 2}^{(l+1 / 2)}=\tilde{\Phi}_{1, J+1 / 2}^{(l+1 / 2)}, \\
& 0=\frac{1}{J} \sum_{j=1}^{J} \tilde{\Phi}_{j}^{(l+1)}
\end{aligned}
$$

and

$$
\begin{aligned}
\tilde{\phi}^{(l+1)}(x)=\tilde{\phi}^{(l+1 / 2)}(x)+ & \tilde{\Phi}_{j}^{(l+1)}-\tilde{\Phi}_{j}^{(l+1 / 2)}, \\
& x_{j-1 / 2} \leq x \leq x_{j+1 / 2}, \quad 1 \leq j \leq J .
\end{aligned}
$$

We note that several of the linearized equations differ in form from Eqs. (2) (6), because the original equations contained nonlinear terms.

After some straightforward algebra (omitted here for brevity), we find:

$$
\delta^{(l+1)}=0 .
$$

This shows that the non-random MC-CMFD method converges the $O(\epsilon)$ component of the system eigenvalue after only one iteration. However, the $O(\epsilon)$ component of the eigenfunction is not converged after one iteration; the Fourier analysis predicts the rate of convergence of this component of the eigenfunction.

Inserting Eq. (14) into Eqs. (13a) and rearranging to algebraically eliminate $\hat{d}_{j+1 / 2}^{(l+1 / 2)}$, we obtain the following system of equations for the linearized nonrandom MC-CMFD method:

$$
\begin{aligned}
\mu \frac{d}{d x} \tilde{\psi}^{(l+1 / 2)}(x, \mu)+\Sigma_{t} \tilde{\psi}^{(l+1 / 2)}(x, \mu) & -\frac{\Sigma_{s}}{2} \tilde{\phi}^{(l+1 / 2)}(x)=\frac{\Sigma_{a}}{2} \tilde{\phi}^{(l)}(x), \\
0 & \leq x \leq X,-1 \leq \mu \leq 1, \\
\tilde{\psi}^{(l+1 / 2)}(0, \mu) & =\tilde{\psi}^{(l+1 / 2)}(X, \mu),-1 \leq \mu \leq 1,
\end{aligned}
$$




$$
\begin{aligned}
& \tilde{\phi}^{(l+1 / 2)}(x)=\int_{-1}^{1} \tilde{\psi}^{(l+1 / 2)}(x, \mu) d \mu, \\
& \tilde{\Phi}_{j}^{(l+1 / 2)}=\frac{1}{\Delta} \int_{x_{j-1 / 2}}^{x_{j+1 / 2}} \tilde{\phi}^{(l+1 / 2)}(x) d x, \\
& \tilde{\Phi}_{1, j+1 / 2}^{(l+1 / 2)}=\int_{-1}^{1} \mu \tilde{\psi}^{(l+1 / 2)}\left(x_{j+1 / 2}, \mu\right) d \mu, \\
& \tilde{\Phi}_{j+1}^{(l+1)}-2 \tilde{\Phi}_{j}^{(l+1)}+\tilde{\Phi}_{j-1}^{(l+1)}=\frac{1}{\tilde{D}}\left(\tilde{\Phi}_{1, j+1 / 2}^{(l+1 / 2)}-\tilde{\Phi}_{1, j-1 / 2}^{(l+1 / 2)}\right) \\
& +\tilde{\Phi}_{j+1}^{(l+1 / 2)}-2 \tilde{\Phi}_{j}^{(l+1 / 2)}+\tilde{\Phi}_{j-1}^{(l+1 / 2)}, \quad 1 \leq j \leq J, \\
& \tilde{\Phi}_{1}^{(l+1)}=\tilde{\Phi}_{J+1}^{(l+1)}, \\
& 0=\frac{1}{J} \sum_{j=1}^{J} \tilde{\Phi}_{j}^{(l+1)}, \\
& \tilde{\phi}^{(l+1)}(x)=\tilde{\phi}^{(l+1 / 2)}(x)+\tilde{\Phi}_{j}^{(l+1)}-\tilde{\Phi}_{j}^{(l+1 / 2)}, \\
& x_{j-1 / 2} \leq x \leq x_{j+1 / 2}, \quad 1 \leq j \leq J .
\end{aligned}
$$

In Eqs. (15), the eigenvalue is converged exactly [Eq. (14)], but the eigenfunction is not converged. The Fourier analysis of the linearized equations, performed next in Section 4, determines the rate of convergence of the eigenfunction. We emphasize again that the Fourier analysis contains no terms that account for statistical errors; the analysis applies only when the statistical noise in the solution is sufficiently small that it can be ignored.

\section{FOURIER ANALYSIS}

To carry out the Fourier analysis, we discretize the Monte Carlo portion of the linearized system in angle and space. This is an approximation to the continuous Monte Carlo problem, but it enables us to formulate and solve a block matrix system numerically for the spectral radius. To ensure that the discrete results are accurate enough to closely approximate the original problem, we carry out a parametric study to determine an adequately fine space-angle grid (see Section 5). We note that the Fourier analysis shown in this section considers only the monoenergetic $k$-eigenvalue problem; however, we have also completed a two-energy-group analysis as part of this work. Numerical results for the two-group analysis are presented in Section 5.

Thus, we approximate the Monte Carlo $k$-eigenvalue problem by a discrete ordinates problem formulated on a fine space-angle grid, with $M$ discrete angles from $1 \leq m \leq M$ and $K$ fine spatial cells of thickness $h(1 \leq k \leq K)$. We use the "coarse-grid parameter" $p=\frac{\Delta}{h}$ to denote the number of fine cells per coarse 
cell, and we introduce the notation

$$
\sum_{k \in j}(\cdot)
$$

to describe a sum over the fine cells $k$ belonging to coarse cell $j$. Also, we use Gauss-Legendre quadrature sets, which satisfy:

$$
\sum_{m=1}^{M} w_{m}=2 .
$$

Using this notation, the discretized version of Eq. (15a) can be written in the form:

$$
\begin{array}{r}
\frac{\mu_{m}}{h}\left(\tilde{\psi}_{k+1 / 2, m}^{(l+1 / 2)}-\tilde{\psi}_{k-1 / 2, m}^{(l+1 / 2)}\right)+\Sigma_{t} \tilde{\psi}_{k, m}^{(l+1 / 2)}-\frac{\Sigma_{s}}{2} \tilde{\phi}_{k}^{(l+1 / 2)}=\frac{\Sigma_{a}}{2} \tilde{\phi}_{k}^{(l)}, \\
1 \leq m \leq M, \quad 1 \leq k \leq K, \\
\tilde{\psi}_{1 / 2, m}^{(l+1 / 2)}=\tilde{\psi}_{K+1 / 2, m}^{(l+1 / 2)}, \quad 1 \leq m \leq M .
\end{array}
$$

Similarly, the linearized scalar flux expression becomes:

$$
\tilde{\phi}_{k}^{(l+1 / 2)}=\sum_{m=1}^{M} w_{m} \tilde{\psi}_{k, m}^{(l+1 / 2)} .
$$

To close the system, we introduce the weighted diamond auxiliary equations:

$$
\begin{aligned}
& \tilde{\psi}_{k, m}^{(l+1 / 2)}=\left(\frac{1+\alpha_{m}}{2}\right) \tilde{\psi}_{k+1 / 2, m}^{(l+1 / 2)}+\left(\frac{1-\alpha_{m}}{2}\right) \tilde{\psi}_{k-1 / 2, m}^{(l+1 / 2)}, \\
& 1 \leq m \leq M, 1 \leq k \leq K
\end{aligned}
$$

In this work, we use the Step Characteristic spatial discretization, with

$$
\alpha_{m}=\frac{1+e^{-\Sigma_{t} h / \mu_{m}}}{1-e^{-\Sigma_{t} h / \mu_{m}}}-\frac{2 \mu_{m}}{\Sigma_{t} h}, \quad 1 \leq m \leq M .
$$

The equations that "link" the Monte Carlo problem to CMFD (Eqs. (15d), (15e) and (16)) must also be discretized, while the already-discrete CMFD equations (Eqs. (15f) and (15g)) remain essentially unchanged:

$$
\begin{aligned}
\tilde{\Phi}_{j}^{(l+1 / 2)} & =\frac{1}{p} \sum_{k \in j} \tilde{\phi}_{k}^{(l+1 / 2)}, \\
\tilde{\Phi}_{1, j+1 / 2}^{(l+1 / 2)} & =\sum_{m=1}^{M} w_{m} \mu_{m} \tilde{\psi}_{(j p)+1 / 2, m}^{(l+1 / 2)}, \\
\tilde{\Phi}_{j+1}^{(l+1)}-2 \tilde{\Phi}_{j}^{(l+1)}+\tilde{\Phi}_{j-1}^{(l+1)} & =\frac{1}{\tilde{D}}\left(\tilde{\Phi}_{1, j+1 / 2}^{(l+1 / 2)}-\tilde{\Phi}_{1, j-1 / 2}^{(l+1 / 2)}\right)
\end{aligned}
$$




$$
\begin{aligned}
\quad+\tilde{\Phi}_{j+1}^{(l+1 / 2)}-2 \tilde{\Phi}_{j}^{(l+1 / 2)}+\tilde{\Phi}_{j-1}^{(l+1 / 2)} & \\
\tilde{\Phi}_{1,1 / 2}^{(l+1)} & =\tilde{\Phi}_{1, J+1 / 2}^{(l+1)} \\
0 & =\frac{1}{J} \sum_{j=1}^{J} \tilde{\Phi}_{j}^{(l+1)}, \\
\tilde{\phi}_{k}^{(l+1)} & =\tilde{\phi}_{k}^{(l+1 / 2)}+\tilde{\Phi}_{j}^{(l+1)}-\tilde{\Phi}_{j}^{(l+1 / 2)}, 1 \leq j \leq J, k \in j .
\end{aligned}
$$

To simplify the Fourier ansatz, a new relative coordinate system is introduced [8]:

$$
k=(j-1) p+r .
$$

Here, $k$ is the global fine cell index, $j$ is the index of the coarse cell in which fine cell $k$ resides, $p$ is the number of fine cells per coarse cell, and $r$ is the position of fine cell $k$ within coarse cell $j$. With this coordinate system in place, we introduce the Fourier ansatz:

$$
\begin{aligned}
\tilde{\psi}_{k-1 / 2, m}^{(l+1 / 2)} & =\omega^{l} a_{r, m} e^{i \Sigma_{t} \lambda x_{j}}, \\
\tilde{\psi}_{k, m}^{(l+1 / 2)} & =\omega^{l} b_{r, m} e^{i \Sigma_{t} \lambda x_{j}}, \\
\tilde{\phi}_{k}^{(l+1 / 2)} & =\omega^{l} B_{r} e^{i \Sigma_{t} \lambda x_{j}}, \\
\tilde{\Phi}_{j}^{(l+1 / 2)} & =\omega^{l} \mathcal{D} e^{i \Sigma_{t} \lambda x_{j}}, \\
\tilde{\Phi}_{j}^{(l+1)} & =\omega^{l} F e^{i \Sigma_{t} \lambda x_{j}}, \\
\tilde{\phi}_{k}^{(l+1)} & =\omega^{l+1} G_{r} e^{i \Sigma_{t} \lambda x_{j}} .
\end{aligned}
$$

The fine-grid error coefficients are assumed to be periodic on the coarse grid, such that:

$$
\tilde{\psi}_{(k+p)-1 / 2, m}^{(l+1 / 2)}=\left(\tilde{\psi}_{k-1 / 2, m}^{(l+1 / 2)}\right) e^{i \Sigma_{t} \lambda \Delta}=\omega^{l} a_{r, m} e^{i \Sigma_{t} \lambda\left(x_{j}+\Delta\right)} .
$$

Inserting the ansatz into Eqs. (18) and simplifying, we obtain the following system of equations:

$$
\begin{aligned}
& \begin{cases}\frac{\mu_{m}}{h}\left(a_{r+1, m}-a_{r, m}\right)+\Sigma_{t} b_{r, m}-\frac{\Sigma_{s}}{2} B_{r}=\frac{\Sigma_{a}}{2} G_{r}, & 1 \leq r<p, \\
\frac{\mu_{m}}{h}\left(a_{1, m} e^{i \Sigma_{t} \lambda \Delta}-a_{r, m}\right)+\Sigma_{t} b_{r, m}-\frac{\Sigma_{s}}{2} B_{r}=\frac{\Sigma_{a}}{2} G_{r}, & r=p,\end{cases} \\
& a_{1, m}=a_{1, m} e^{i \Sigma_{t} \lambda X}, \\
& \begin{cases}b_{r, m}=\left[\frac{1+\alpha_{m}}{2}\right] a_{r+1, m}+\left[\frac{1-\alpha_{m}}{2}\right] a_{r, m}, & 1 \leq r<p, \\
b_{r, m}=\left[\frac{1+\alpha_{m}}{2}\right] a_{1, m} e^{i \Sigma_{t} \lambda \Delta}+\left[\frac{1-\alpha_{m}}{2}\right] a_{r, m} & r=p,\end{cases} \\
& B_{r}=\sum_{m=1}^{M} w_{m} b_{r, m}
\end{aligned}
$$




$$
\begin{aligned}
\mathcal{D} & =\frac{1}{p} \sum_{r=1}^{p} B_{r}, \\
2(F-\mathcal{D})\left(\cos \left(\Sigma_{t} \lambda \Delta\right)-1\right) & =3 \Sigma_{t} \Delta \sum_{m=1}^{M} w_{m} \mu_{m} a_{1, m}\left(e^{i \Sigma_{t} \lambda \Delta}-1\right), \\
\sum_{m=1}^{M} w_{m} \mu_{m} a_{1, m} & =e^{i \Sigma_{t} \lambda X} \sum_{m=1}^{M} w_{m} \mu_{m} a_{1, m}, \\
0 & =\sum_{j=1}^{J} e^{i \Sigma_{t} \lambda x_{j}}, \\
\omega G_{r}-B_{r} & =(F-\mathcal{D}) .
\end{aligned}
$$

From the boundary equations (22b) and (22g), we determine permissible values of the Fourier frequency $\lambda$ for the discrete system:

$$
\lambda_{n}=\frac{2 n \pi}{\Sigma_{t} X}, 1 \leq n<J .
$$

This set of Fourier frequencies automatically satisfies the normalization condition in Eq. (22h). Next, Eqs. (22d) and (22i) are used to eliminate $F, \mathcal{D}$, and $B_{r}$ in the remainder of Eqs. (22). We also define

$$
c=\frac{\Sigma_{s}}{\Sigma_{t}} .
$$

Inserting Eq. (24) and carrying out further simplification yields the following discrete system of equations:

$$
\begin{gathered}
\left\{\begin{array}{cc}
\frac{\mu_{m}}{\Sigma_{t} h}\left(a_{r+1, m}-a_{r, m}\right)+b_{r, m}-\frac{c}{2} \sum_{m^{\prime}=1}^{M} w_{m^{\prime}} b_{r, m^{\prime}}-\frac{(1-c)}{2} G_{r}=0 \\
\frac{\mu_{m}}{\Sigma_{t} h}\left(a_{1, m} e^{i \Sigma_{t} \lambda_{n} \Delta}-a_{r, m}\right)+b_{r, m}-\frac{c}{2} \sum_{m^{\prime}=1}^{M} w_{m^{\prime}} b_{r, m^{\prime}}-\frac{(1-c)}{2} G_{r}=0 \\
r=p
\end{array}\right. \\
\left\{\begin{array}{cc}
{\left[\frac{1+\alpha_{m}}{2}\right] a_{r+1, m}+\left[\frac{1-\alpha_{m}}{2}\right] a_{r, m}-b_{r, m}=0,} & 1 \leq r<p, \\
{\left[\frac{1+\alpha_{m}}{2}\right] a_{1, m} e^{i \Sigma_{t} \lambda_{n} \Delta}+\left[\frac{1-\alpha_{m}}{2}\right] a_{r, m}-b_{r, m}=0} & r=p,
\end{array}\right. \\
\sum_{m=1}^{M} w_{m}\left(E \mu_{m} a_{1, m}+b_{r, m}\right)-\omega G_{r}=0
\end{gathered}
$$

where

$$
E=\frac{3 \Sigma_{t} \Delta}{2}\left[1+\frac{i \sin \left(\Sigma_{t} \lambda_{n} \Delta\right)}{\left(\cos \left(\Sigma_{t} \lambda_{n} \Delta\right)-1\right)}\right] .
$$


Eqs. (25) can be written in block matrix form [8]:

$$
\left[\begin{array}{cc}
\mathbf{A} & \mathbf{B} \\
\mathbf{C} & -\omega \mathbf{I}
\end{array}\right]\left[\begin{array}{l}
\mathbf{x} \\
\mathbf{y}
\end{array}\right]=\left[\begin{array}{l}
\mathbf{0} \\
\mathbf{0}
\end{array}\right]
$$

Here $\mathbf{A}$ is a $2 M p \times 2 M p$ matrix containing the coefficients of $a_{r, m}$ and $b_{r, m}$ from Eqs. (25a) and (25b), B is a $2 M p \times p$ matrix composed of the coefficients of $G_{r}$ from Eqs. (25a) and (25b), and $\mathbf{C}$ is a $p \times 2 M p$ matrix containing the coefficients of $a_{r, m}$ and $b_{r, m}$ from Eq. (25c). The final matrix $(-\omega \mathbf{I})$ is a $p \times p$ diagonal matrix containing the negative of the eigenvalues $\omega_{r}$, which comprise the coefficients of $G_{r}$ in Eqs. (25c). The size- $2 M p$ column vector $\mathbf{x}$ contains the $a_{r, m}$ and $b_{r, m}$ values, while the size- $p$ column vector $\mathbf{y}$ contains the $G_{r}$ values. To solve for the system eigenvalues $\omega_{r}$, we follow the procedure outlined in [8]. The block matrix system is first decomposed:

$$
\left[\begin{array}{cc}
\mathbf{A} & \mathbf{B} \\
\mathbf{C} & -\omega \mathbf{I}
\end{array}\right]=\left[\begin{array}{ll}
\mathbf{A} & \mathbf{0} \\
\mathbf{C} & \mathbf{I}
\end{array}\right]\left[\begin{array}{cc}
\mathbf{I} & \mathbf{A}^{-1} \mathbf{B} \\
\mathbf{0} & -\mathbf{C A}^{-1} \mathbf{B}-\omega \mathbf{I}
\end{array}\right] .
$$

The determinant of the original system is then calculated using

$$
\left|\begin{array}{ll}
\mathbf{A} & \mathbf{0} \\
\mathbf{C} & \mathbf{I}
\end{array}\right|\left|\begin{array}{cc}
\mathbf{I} & \mathbf{A}^{-1} \mathbf{B} \\
\mathbf{0} & -\mathbf{C A}^{-1} \mathbf{B}-\omega \mathbf{I}
\end{array}\right|=\left|\mathbf{A} \|-\mathbf{C A}^{-1} \mathbf{B}-\omega \mathbf{I}\right|=\mathbf{0} .
$$

From inspection, the determinant of the original block matrix system is zero, while the determinant of matrix $\mathbf{A}$ is non-zero. Thus, we can infer that

$$
\left|-\mathbf{C A}^{-1} \mathbf{B}-\omega \mathbf{I}\right|=\mathbf{0},
$$

where $\mathbf{C A}^{-\mathbf{1}} \mathbf{B}$ and $\mathbf{I}$ are $p \times p$ matrices.

Using Eq. (29), the eigenvalues $\omega_{r}$ can be calculated numerically for $1 \leq r \leq p$ and permitted values of the discrete Fourier frequency, $\lambda_{n}$. Once the eigenvalues are known, the spectral radius $\rho$ is determined using

$$
\rho=\sup _{1 \leq n<J}\left(\sup _{1 \leq r \leq p}\left|\omega_{r}\left(\lambda_{n}\right)\right|\right) .
$$

\section{APPLICATION TO MC-CMFD AND $S_{N}$ SURROGATE}

As a first test of the validity of the Fourier analysis, we compare its predictions to spectral radius estimates obtained from a "surrogate" discrete ordinates - CMFD $\left(\mathrm{S}_{\mathrm{N}}-\mathrm{CMFD}\right)$ code. The surrogate code treats the scattering source implicitly, as in the non-random MC-CMFD method. Instead of performing a fixed number of inner scattering source iterations per outer fission source iteration, the surrogate code performs as many inner scattering source iterations as is necessary to converge the scattering source to a very small tolerance. When run on a fine space-angle grid, the surrogate code should behave much like Monte Carlo in the limit as the number of histories approaches infinity. Thus, the spectral 
radius estimate produced using a fine space-angle grid should well-approximate the estimate using the Fourier analysis.

To determine a sufficiently fine space-angle grid, we performed a parametric study using the monoenergetic Fourier analysis script developed for this work. Values of the coarse-grid parameter $p$ and angular order $M$ were successively increased for a monoenergetic problem with fixed cross sections $\Sigma_{t}=1 \mathrm{~cm}^{-1}$, $\nu \Sigma_{f}=0.01 \mathrm{~cm}^{-1}$, and $\Sigma_{s}=0.5 \mathrm{~cm}^{-1}$. The study was repeated twice, for coarse grids $\Delta=1 \mathrm{~cm}$ and $\Delta=5 \mathrm{~cm}$. Resulting spectral radius estimates were then plotted as a function of the coarse-grid parameter and number of discrete angles. These plots are shown in Fig. (1).

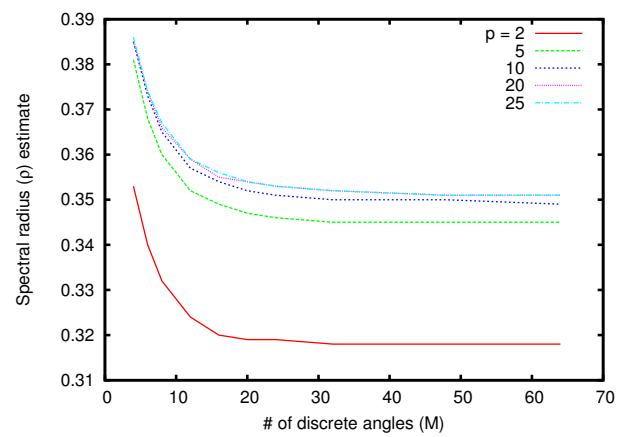

(a) $\Delta=1 \mathrm{~cm}$

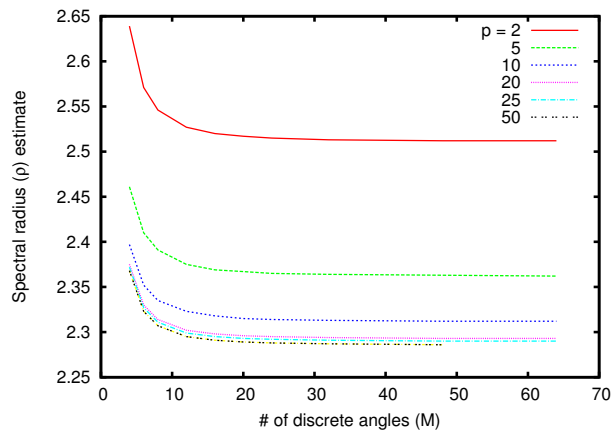

(b) $\Delta=5 \mathrm{~cm}$

Figure 1: Discrete spectral radius estimate $(\rho)$ as a function of angular order $M$ and coarse-grid parameter $p$

In both cases, the spectral radius curve changes significantly when the number of discrete angles $M$ is small, but becomes flat for $M \geq 16$. For the $\Delta=1$ $\mathrm{cm}$ case, we see a large shift in the spectral radius estimate between the $p=2$ and $p=5$ curves, but for $p \geq 10$ the change in the spectral radius is small (on the order of $\left.10^{-3}\right)$. For $\Delta=5 \mathrm{~cm}$, the change in the spectral radius estimate becomes small for $p \geq 25$.

Based on this study, we opted to use $p=50$ and the $\mathrm{S}_{32}$ quadrature set for our monoenergetic simulations, and $p=50$ with the $\mathrm{S}_{16}$ quadrature set for our two-group simulations. These discretizations provide sufficient accuracy for the discrete approximation across a wide range of coarse grid sizes, while maintaining reasonable runtimes and memory demands for the Fourier analysis script. Due to the size of the block matrix system $([2 M p+p] \times[2 M p+p]$ for monoenergetic problems, and $[2 M p+2(p+1)] \times[2 M p+2(p+1)]$ for two-group problems), the spectral radius calculation becomes intractable for large values of $M$ and $p$. While there is inevitably an error in the discrete results, this error should be small.

Next, in our direct MC-CMFD and surrogate $\mathrm{S}_{\mathrm{N}}-\mathrm{CMFD}$ simulations, we 
employed the following well-known spectral radius estimate:

$$
\rho=\frac{\left\|\Phi^{(l+1)}-\Phi^{(l)}\right\|}{\left\|\Phi^{(l)}-\Phi^{(l-1)}\right\|} .
$$

This expression requires estimates of $\Phi$ for three consecutive iterations, and is accurate for large values of $l$. For small values of $\rho$, this is slightly problematic for the surrogate $\mathrm{S}_{\mathrm{N}}$-CMFD simulations, since roundoff errors can significantly affect the accuracy of Eq. (31) after only a few iterations. It is much more problematic for the random MC-CMFD simulations, since the error in the fission source can quickly become reduced to the magnitude of the statistical errors.

Fortunately, small values of $\rho$, which are the most difficult to estimate, are not so interesting because the iteration method converges very rapidly. Larger values of $\rho$, closer to or greater than unity, are much more interesting, and for these Eq. (31) is more accurate.

We generate numerical results for both monoenergetic and two-group $k$ eigenvalue problems. The monoenergetic analysis allows us to determine straightforward relationships between coarse grid optical thickness and spectral radius, while the two-group results provide spectral radius estimates for more "realistic" cross section data. In Section 5.1, we present numerical results for the monoenergetic analysis.

\subsection{Monoenergetic Case}

The monoenergetic $k$-eigenvalue test problem considered in this paper is a homogeneous 50-centimeter slab with periodic boundaries. The total and fission cross sections are fixed, while the scattering cross section takes one of four values. The coarse grid size varies between 1.0 and 12.5 mean free paths (mfp), keeping in mind that there must be an integer number of coarse cells. Numerical data for the problem are provided in Table 1 .

Table 1: Monoenergetic Test Problem Specifications

\begin{tabular}{||c|c|c|c||}
\hline \hline$\Sigma_{t}\left(\mathrm{~cm}^{-1}\right)$ & $\nu \Sigma_{f}\left(\mathrm{~cm}^{-1}\right)$ & $\Sigma_{s}\left(\mathrm{~cm}^{-1}\right)$ & $\Delta(\mathrm{cm})$ \\
\hline 1.0 & 0.01 & $0.5,0.75,0.9,0.99$ & $1.0-12.5$ \\
\hline \hline
\end{tabular}

For each coarse grid/scattering ratio combination, we calculate the theoretical spectral radius using $p=50$ with the $S_{32}$ Gauss-Legendre quadrature set. The numerical spectral radius is then estimated using our surrogate $\mathrm{S}_{\mathrm{N}}-\mathrm{CMFD}$ code. We reiterate that the discrete ordinates code performs many sweeps (inner iterations) to converge the scattering source to a tight tolerance $\left(10^{-9}\right)$ before proceeding to the CMFD calculation, in order to mimic the non-random MC-CMFD method.

We were also able to estimate the spectral radius directly using a MC-CMFD code. Unfortunately, it is difficult to estimate $\rho$ when the spectral radius is small (i.e. the fission source converges very quickly), because the magnitude of the 
fission source error becomes comparable to the stochastic error after very few cycles. (To minimize this stochastic error, we employ track-length estimators for all of our monoenergetic simulations.) Eq. (31) requires three cycles of flux data to numerically estimate the spectral radius; if $\rho$ is small, the fission source error may already be small compared to the random error before an estimate can be calculated! Difficulties also occur when $\rho$ is less than but close to unity; for these problems the random statistical errors in MC-CMFD can drive the barely-stable non-random MC-CMFD method unstable.

To estimate the MC-CMFD spectral radius numerically, the code was initialized with a random fission source guess. We then used Eq. (31) to generate a single value of $\rho$ after the fourth cycle. This procedure was repeated for twentyfive independent simulations to obtain an average MC-CMFD $\rho$ value, which is plotted along with its standard deviation in the subsequent figures. Evidence from multiple trials shows that reasonably accurate estimates of $\rho$ can only be obtained during the first few inactive cycles of each simulation (when the fission source error is larger than the random error). Because three cycles of coarse-grid fluxes are required to generate an estimate of $\rho$, the first value is not available until the end of the third iteration cycle. This estimate tends to be slightly biased (likely due to contamination from the initial guess), and we discard it. As a result, the earliest usable spectral radius estimate is calculated after the fourth iteration cycle.

In spite of these difficulties, we were able to calculate direct MC-CMFD spectral radius estimates for problems in which $0.5<\rho<0.98$. Again, if $\rho<0.5$, the fission source converges too rapidly for Eq. (31) to become accurate. Also, if $\rho>0.98$, then the random statistical errors in MC-CMFD can drive the barely-stable non-random MC-CMFD method unstable. For this reason, our direct estimates of $\rho$ from MC-CMFD simulations become very noisy when $\rho \approx 1$.

But perhaps most importantly, our direct numerical simulations show that for a given scattering ratio, the Fourier analysis accurately predicts the coarse grid size at which the simulation becomes unstable.

Figs. (2) through (5) compare the theoretical, $\mathrm{S}_{N}$-CMFD and MC-CMFD spectral radius results for $c=0.5,0.75,0.9$ and 0.99 , respectively. The $\mathrm{S}_{N^{-}}$ CMFD spectral radius very closely matches the theoretical prediction for most cases, with the exception of problems in which the true spectral radius is very low. In these cases, the $\mathrm{S}_{N^{-}}$CMFD solution converges to machine precision too quickly to obtain an asymptotic $\rho$ estimate.

There are several interesting trends in the MC-CMFD spectral radius data. First, the spectral radius increases monotonically as the coarse grid size increases. There appear to no be no coarse grid sizes between $\Delta=1 \mathrm{mfp}$ and $\Delta=12.5 \mathrm{mfp}$ for which the MC-CMFD method is stable for all values of the scattering ratio $0 \leq c<1$, even in the limit as the number of particles becomes infinite.

Also, the Fourier analysis predicts that for a fixed coarse grid size, the spectral radius decreases (the method converges more rapidly) as the scattering ratio increases. This trend can be explained by examination of the MC-CMFD 


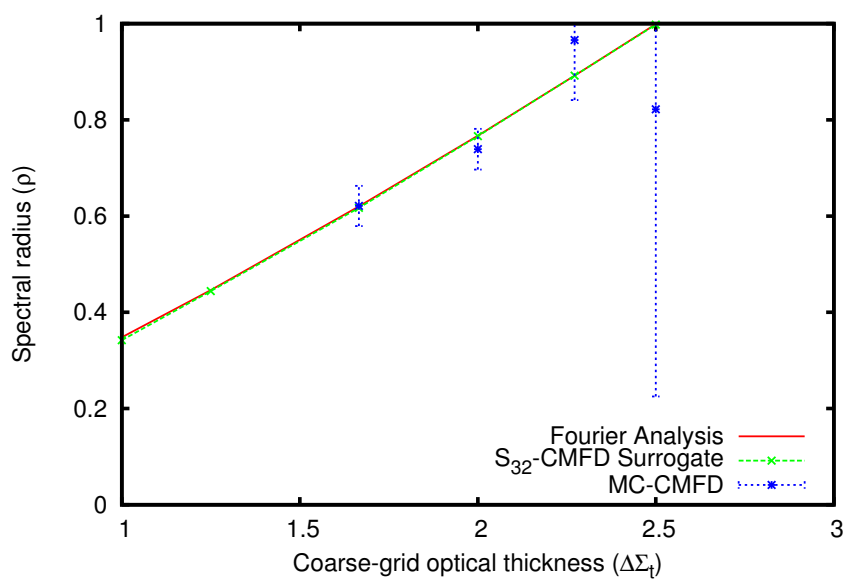

Figure 2: Spectral radius $(\rho)$ vs. coarse-grid optical thickness for $c=0.5$

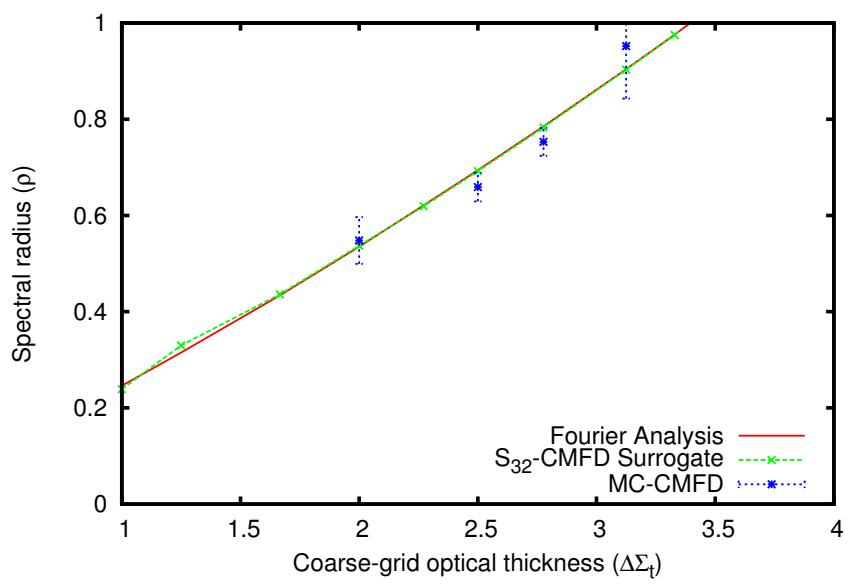

Figure 3: Spectral radius $(\rho)$ vs. coarse-grid optical thickness for $c=0.75$

iteration strategy. As previously described, the Monte Carlo method treats scattering implicitly; the scattering source is converged entirely during the Monte Carlo sweep. If the scattering ratio is near unity, the Monte Carlo simulation does more "work" during a single iteration than it would for a low-scattering problem. This transfers a considerable amount of the convergence burden to the inner Monte Carlo sweep, which results in outer (CMFD) fission source convergence after very few cycles.

In addition, Figs. (2) - (5) show that the standard deviation of the MCCMFD spectral radius estimate becomes large when $\rho \approx 1$. As the theoretical spectral radius approaches unity, the fission source convergence rate becomes ar- 


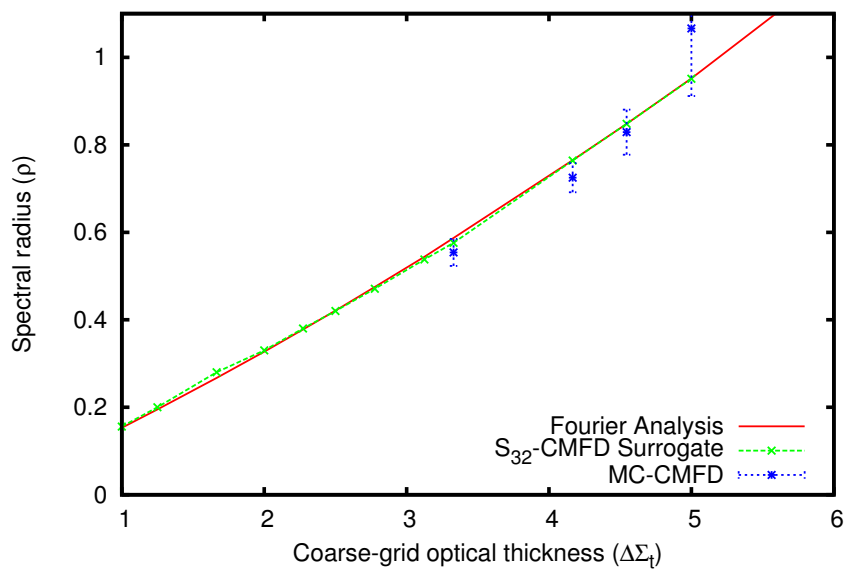

Figure 4: Spectral radius $(\rho)$ vs. coarse-grid optical thickness for $c=0.9$

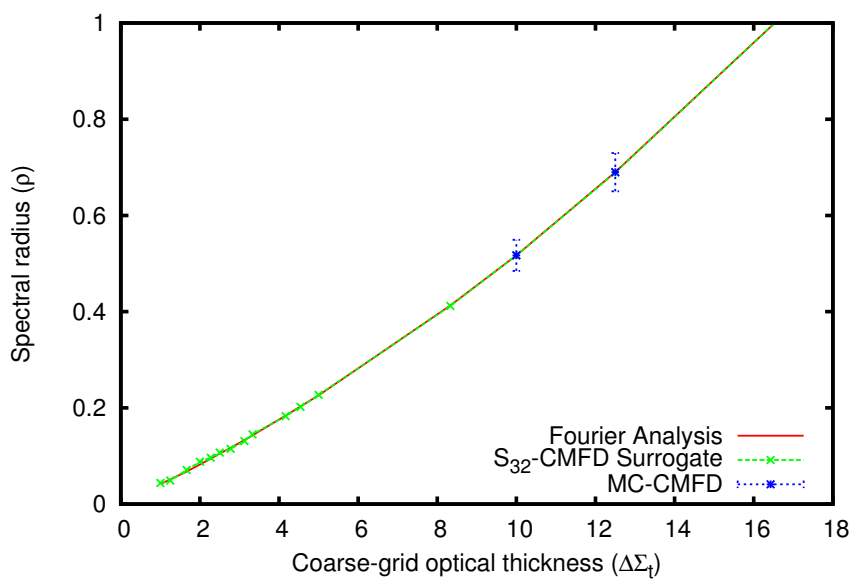

Figure 5: Spectral radius $(\rho)$ vs. coarse-grid optical thickness for $c=0.99$

bitrarily slow for the hypothetical case with no stochastic noise. Since our Monte Carlo simulations are necessarily run with a finite number of particles per cycle, our simulations have a non-negligible random error in quantities tallied during the Monte Carlo sweep. This additional error term can induce an instability in problems that are theoretically stable (in the infinite-particle limit). Therefore, spectral radius estimates from our MC-CMFD code, for problems very near the predicted stability limit, tend to be noisy. This is especially obvious for the $c=0.5$ case in Fig. (2).

When the MC-CMFD method is used in an unstable configuration, the coarse-grid flux shape develops a distinct cycle-to-cycle oscillation. An example 
of this is given in Fig. (6a), with a stable simulation in Fig. (6b) for comparison. The even-cycle fluxes in Fig. (6a) become increasingly tipped toward the left edge of the slab as the iteration cycles progress, while the odd-cycle fluxes increasingly tip toward the right. Eventually, the simulation will crash when the coarse-grid flux becomes negative, or when one or more coarse cells have no Monte Carlo data (we disabled negative flux detection to generate data for Fig

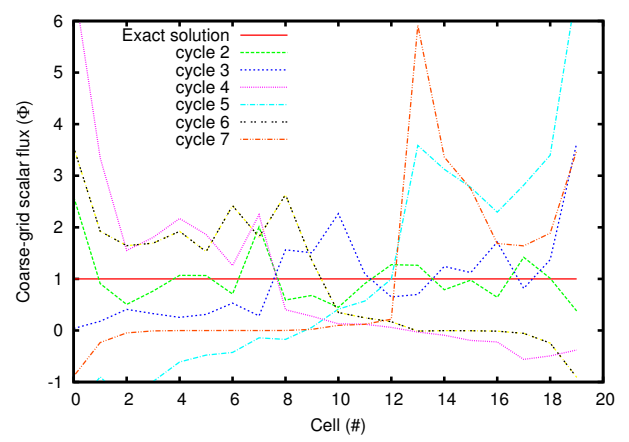

(a) $\Sigma_{t} \Delta=2.5$

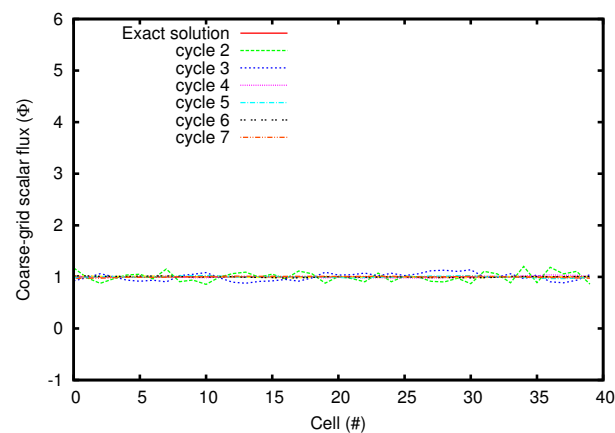

(b) $\Sigma_{t} \Delta=1.25$

Figure 6: Unstable vs. stable cycle-to-cycle coarse grid fluxes, $c=0.5$

(6a)). We stress that the only difference between the results presented in Fig. (6a) and Fig. (6b) is the coarse grid size; both simulations use the same random number seed, the same initial guess, the same number of particles per cycle, and the same cross sections. Also, while the example problem shown in Figure 6 is a homogeneous, monoenergetic slab system, analogous oscillating instabilities have been observed in MC-CMFD simulations of multidimensional, multigroup problems with spatial heterogeneity [12].

Recent work [5] has attempted to mitigate oscillation in the MC-CMFD method by using Monte Carlo tallies from multiple cycles to form the CMFD system, a technique known as "accumulation." The effect of this approach is similar to running a larger number of Monte Carlo particles per iteration cycle. However, using tallies from more than one Monte Carlo cycle reintroduces some of the inter-cycle correlation normally suppressed by CMFD feedback. This complicates the iteration strategy by creating a need to reset Monte Carlo tallies periodically throughout the calculation, and may bias the solution if CMFD feedback is applied during active cycles. In essence, the tally accumulation technique does not address the underlying cause of the flux oscillation - a coarse grid size near or beyond the stability limit of the MC-CMFD method.

Finally, to better visualize the stability limit of the MC-CMFD method, we interpolated linearly between our Fourier analysis results to produce approximate constant- $\rho$ curves. These curves are plotted as a function of coarse mesh size and scattering ratio, and are shown in Fig. (7). The phase space to the left of the $\rho=1.0$ line represents coarse grid/scattering ratio combinations for which the one-group planar non-random MC-CMFD method is stable. Conversely, 


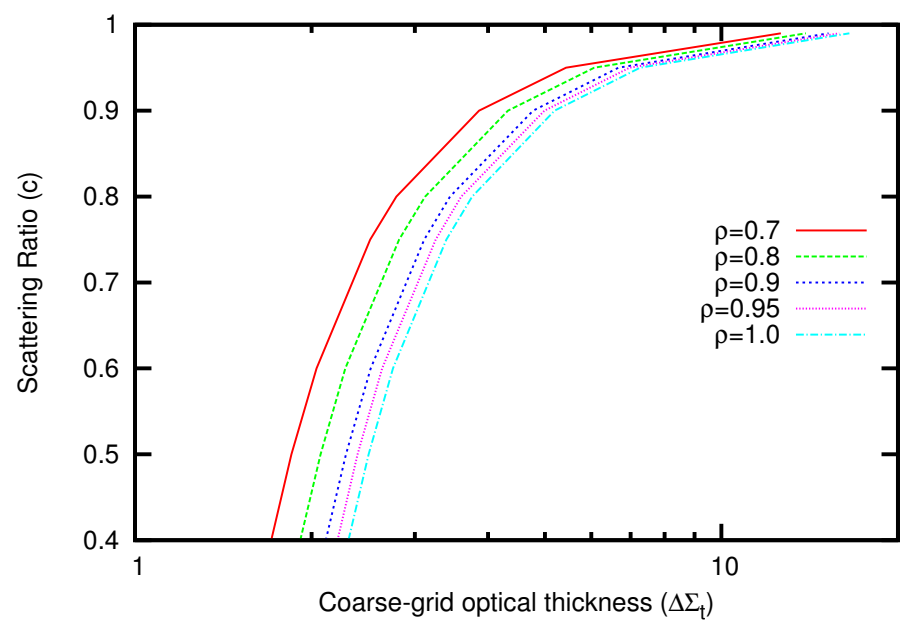

Figure 7: Constant spectral radius curves as a function of $\Sigma_{t} \Delta$ and $c$

the phase space to the right of the $\rho=1.0$ line represents coarse grid/scattering ratio combinations for which the method is unstable. As the scattering ratio approaches unity, the non-random MC-CMFD stable grid size limit appears to tend towards infinity; for low scattering ratios $(c \leq 0.5)$, the method becomes unstable for grids on the order of two mean free paths. The presence of random error probably shifts these curves slightly to the left, but we cannot quantify this effect at present.

Next, we present spectral radius results for the two-group Fourier analysis, and compare them to numerical spectral radius estimates from MC-CMFD and surrogate $\mathrm{S}_{N^{-}}$CMFD simulations.

\subsection{Two-group Case}

In this section, we present two-group numerical spectral radius results for a homogeneous $\mathrm{UO}_{2}$ fuel problem, and compare these results to theoretical predictions from our two-group Fourier analysis. To simplify the implementation of the Fourier analysis, we have assumed that (i) upscattering from the thermal group to the fast group is negligible, (ii) all fission neutrons are born in the fast group, and (iii) fast fission is negligible. Mathematical details of the two-group analysis are omitted from this paper for brevity, but can be found in [12].

Our two-group test problem uses $\mathrm{UO}_{2}$ material cross-sections from the C5G7 benchmark [13]; however, because the C5G7 benchmark specification provides seven-group cross sections, we first flux-collapse the original cross sections to two energy groups using a discrete-ordinates code (for a homogeneous infinitemedium system). Using our Fourier analysis script, a small energy group parametric was performed to investigate the relationship between the choice of energy bin bounds for the two-group collapse and the theoretical spectral radius 
of the MC-CMFD iteration scheme; this study showed very little change in the spectral radius as the group structure changed.

Given this information, we decided to collapse C5G7 groups 1 through 3 into the fast group, with groups 4 through 7 in the thermal group. This structure was chosen with the $\mathrm{MC}$ calculation in mind; in the interest of maintaining fairly comparable $\mathrm{MC}$ statistics between the fast and thermal groups, it is logical to include multiple fine energy groups in the thermal coarse group. In addition, this particular group structure satisfies the no-upscattering assumption made in the two-group Fourier analysis. However, we note that the other two assumptions are violated: the $\mathrm{UO}_{2}$ fast fission cross-section is non-zero, and a small fraction of fission neutrons are born in the thermal group. Table 2 shows the infinite-

Table 2: Two-group $\mathrm{UO}_{2}$ test problem specifications (all cross sections in $\mathrm{cm}^{-1}$ )

\begin{tabular}{||c|c|c|c|c||}
\hline \hline$\Sigma_{t, 1}$ & $\Sigma_{t, 2}$ & $\nu \Sigma_{f, 1}$ & $\nu \Sigma_{f, 2}$ & $\Sigma_{s, 1 \rightarrow 1}$ \\
\hline 0.328 & 0.524 & $3.81 \mathrm{e}-3$ & $4.67 \mathrm{e}-2$ & 0.323 \\
\hline \hline
\end{tabular}

\begin{tabular}{||c|c|c|c|c||}
\hline \hline$\Sigma_{s, 1 \rightarrow 2}$ & $\Sigma_{s, 2 \rightarrow 2}$ & $\chi_{1}$ & $\chi_{2}$ & $\Delta(\mathrm{cm})$ \\
\hline $1.32 \mathrm{e}-4$ & 0.435 & $\sim 1$ & $1.17 \mathrm{e}-7$ & $5.0-37.5$ \\
\hline \hline
\end{tabular}

medium flux-collapsed two-group cross sections for $\mathrm{C} 5 \mathrm{G} 7 \mathrm{UO}_{2}$ fuel, as well as the range of coarse grid sizes considered for our test problem. The use of C5G7 cross sections allows us to investigate the spectral radius as a function of coarse grid size for a realistic reactor material, in the hope that greater insight can be gained regarding the stability behavior of practical MC-CMFD reactor simulations.

Here, we consider a $150-\mathrm{cm}$ slab problem with periodic boundaries. This problem is physically larger than the monoenergetic test problem, because total cross sections for the two-group case are considerably smaller than the monoenergetic total cross section of unity. While the $50-\mathrm{cm}$ monoenergetic test problem was 50 mean free paths (mfp) thick, the 150-cm two-group system is $\sim 49 \mathrm{mfp}$ thick for fast neutrons, and $\sim 79 \mathrm{mfp}$ thick for thermal neutrons.

Because the two-group cross sections involve a scattering matrix, we can no longer derive straightforward relationships between the spectral radius and the scattering ratio. However, we can still investigate the relationship between the spectral radius and coarse grid size.

As was done in the monoenergetic case, we generate numerical spectral radius estimates using a surrogate multigroup $\mathrm{S}_{N^{-}}$CMFD code. Two-group $\mathrm{S}_{N^{-}}$ CMFD cases are simulated using a fine space-angle grid, with $\mathrm{S}_{16}$ quadrature and $\mathrm{p}=50$ fine cells per coarse cell. Ideally, we would hold the fine grid size fixed, rather than the number of fine cells per coarse; however, because the size of the matrix system depends on $\mathrm{p}$, the theoretical spectral radius calculation quickly becomes intractable when the coarse grid size increases and the fine grid 
size is held fixed.

We also generate numerical two-group spectral radius estimates using direct MC-CMFD simulations. As mentioned in Section 5.1, it is difficult to efficiently obtain estimates of the spectral radius using MC-CMFD. If the non-random spectral radius is too low $(<\sim 0.5)$, the fission source converges before we can obtain an accurate estimate. Likewise, if the non-random spectral radius is sufficiently close to unity, statistical errors can push the simulation into the unstable regime; this, in turn, causes the simulation to crash. Finally, a large number of particles per cycle must be simulated to minimize stochastic noise, and each case must be repeated 25 times (using different random number seeds) to estimate error bounds.

These issues are only exacerbated in the two-group case, where the number of tally bins is doubled. In addition, standard track-length estimators cannot be used to compute quantities that depend on post-collision information, so our multigroup simulations must use collision estimators to calculate coarsegrid quantities. Combined, these factors cause direct two-group MC-CMFD spectral radius estimates to be considerably noisier than their monoenergetic counterparts.

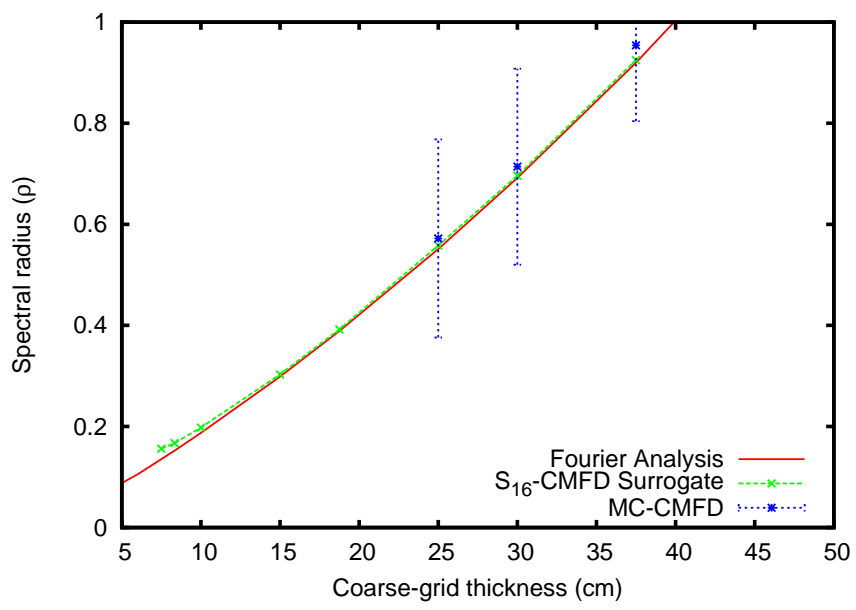

Figure 8: Spectral radius $(\rho)$ vs. coarse-grid thickness, group-collapsed $\mathrm{UO}_{2}$

Figure 8 plots spectral radius versus coarse grid size for the two-group $\mathrm{UO}_{2}$ fuel material group-collapsed from the C5G7 cross-sections. In addition to theoretical predictions from the Fourier analysis, Figure 8 includes numerical estimates from direct MC-CMFD, as well as the $\mathrm{S}_{N}$-CMFD surrogate.

In spite of the aforementioned difficulties, we observe very good agreement between theoretical predictions and surrogate $\mathrm{S}_{N}$-CMFD estimates. In addition, mean MC-CMFD estimates are very close to theoretical predictions; however, standard deviations for these estimates are large, so we cannot draw definitive conclusions from this data. The two-group $\mathrm{UO}_{2}$ spectral radius is a monoton- 
ically increasing function of the coarse grid size, $\Delta$. The homogeneous $150-\mathrm{cm}$ $\mathrm{UO}_{2}$ problem becomes unstable when the coarse grid is roughly $40 \mathrm{~cm}$ thick, which corresponds to an optical thickness of roughly $13 \mathrm{mfp}$ for fast neutrons and $21 \mathrm{mfp}$ for thermal neutrons.

We also investigate a group-collapse case, where the $\mathrm{MC}$ calculation takes place on a more detailed energy grid than the two-group CMFD calculation. To do this, we use the original seven-group C5G7 material cross sections for the $\mathrm{MC}$ (or surrogate $\mathrm{S}_{N}$ ) transport solve. Coarse-grid quantities are tallied according to the coarse group structure introduced earlier in Section 5, resulting in a two-group CMFD system. We have not Fourier-analyzed this scenario, but

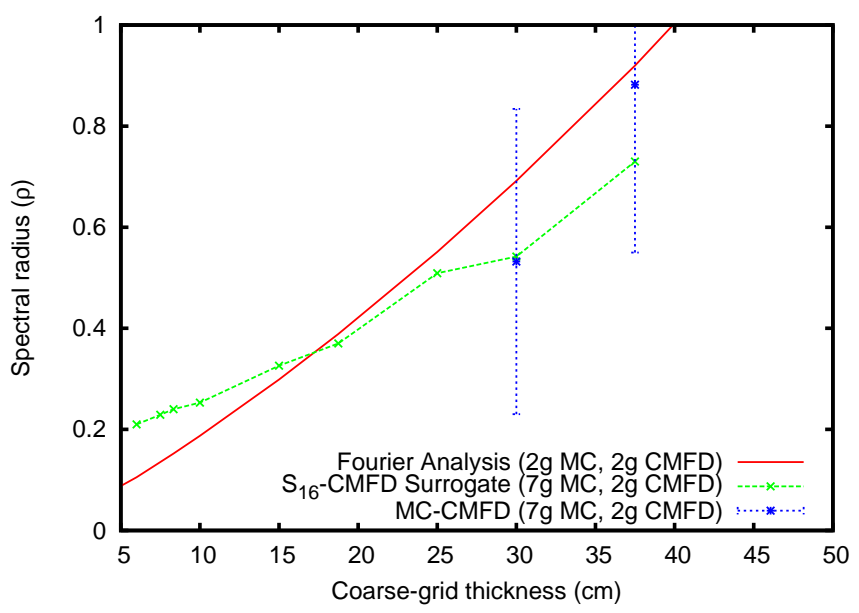

Figure 9: Spectral radius ( $\rho$ ) vs. coarse-grid thickness, 7-group MC/2-group CMFD, $\mathrm{UO}_{2}$

we seek to understand how well the two- group Fourier analysis predicts the convergence rate of the seven-group MC/two-group CMFD iteration scheme (hereafter referred to as the $7 \mathrm{~g} / 2 \mathrm{~g}$ iteration). In the limit as the number of $\mathrm{MC}$ energy groups approaches infinity, the multigroup iteration scheme approaches the continuous-energy MC/two-group CMFD (CE/2g) iteration scheme. Thus, understanding how well our two-group Fourier analysis predicts the stability of the $7 \mathrm{~g} / 2 \mathrm{~g}$ iteration should yield insight into how well it might predict the stability behavior of $\mathrm{CE} / 2 \mathrm{~g}$ simulations.

Figure 9 plots $\mathrm{S}_{N}$-CMFD and MC-CMFD estimates of the spectral radius for the $7 \mathrm{~g} / 2 \mathrm{~g}$ iteration scheme, as a function of the coarse grid size. The theoretical two-group spectral radius curve is included for comparison.

While both the $7 \mathrm{~g} / 2 \mathrm{~g}$ and two-group spectral radius trends are monotonically increasing, it is apparent that the $7 \mathrm{~g} / 2 \mathrm{~g} \mathrm{~S} \mathrm{~S}_{16}$-CMFD surrogate spectral radius curve is flatter than the two-group theoretical curve. In other words, the seven-group/two-group MC-CMFD iteration scheme will likely remain stable for larger coarse grids, in the infinite-particle limit (direct MC-CMFD results presented in Figure 9 are too noisy to provide any further insight). This sug- 
gests that the use of a larger number of energy groups in the MC simulation has a stabilizing effect on the iteration scheme as a whole, even though the coarse-grid optical thickness may be beyond the corresponding monoenergetic stability limit for some energy groups.

For instance, if we use the monoenergetic Fourier analysis to estimate the coarse-grid stability limit for the two-group $\mathrm{UO}_{2}$ problem (using the thermalgroup scattering ratio, $c \approx 0.83$ ), we predict that the two-group simulation will become unstable when the coarse grid is between 3.5 and $5.5 \mathrm{mfp}$ thick (the coarse-grid stability limits for the monoenergetic $c=0.75$ and $c=0.9$ cases, respectively). However, Figure 8 shows that the two-group $\mathrm{UO}_{2}$ simulation remains stable for coarse grids up to $\sim 40 \mathrm{~cm}$ thick, which corresponds to a thermal group optical thickness of roughly $21 \mathrm{mfp}$ - far beyond the monoenergetic stability limit.

This finding may help to explain previously published MC-CMFD results. For example, Herman et. al. [14] have shown that assembly-grid CMFD can be used to accelerate MC fission source convergence for a continuous-energy reactor core problem. However, in [12], assembly-grid CMFD acceleration is shown to be unstable for the seven-group C5G7 benchmark. This difference in stability behavior may be due in part to the differing energy treatment of the two problems; while the C5G7 cross sections are provided on a relatively coarse energy grid, the continuous-energy problem in [14] effectively represents the limit as the number of energy groups becomes infinite. Thus, the stabilizing effect of a refined energy group structure may explain the improved stability characteristics of the continuous-energy problem over the seven-group problem.

\section{DISCUSSION}

In this work, a Fourier analysis is employed to predict the stability and convergence behavior of the MC-CMFD $k$-eigenvalue method, in the limit as the number of Monte Carlo particles approaches infinity. The Fourier analysis procedure is performed for both a monoenergetic system and a simplified twoenergy-group system. To carry out this analysis, (i) a sufficiently large number of Monte Carlo particles is used per cycle that random statistical effects can be ignored, (ii) the non-linear iteration scheme is linearized for a problem having a simple eigenfunction solution, and (iii) the linearized Monte Carlo problem is approximated with discrete ordinates on a fine space-angle grid, making it possible to solve a matrix system numerically for the spectral radius.

Monoenergetic theoretical predictions from the Fourier analysis compare very favorably with numerical results generated by a $\mathrm{S}_{N}$-CMFD surrogate code, and also with a MC-CMFD code - provided that a large number of particles per cycle is used and the predicted spectral radius is not too small. When the predicted spectral radius is small $(\rho<0.5)$, the fission source converges too quickly to obtain an accurate estimate. However, the most interesting situations are ones in which the spectral radius $\rho$ is close to or greater than unity. For these situations, the predictions of our monoenergetic Fourier analysis agree well with direct simulations. 
We also consider a two-group $\mathrm{UO}_{2}$ fuel problem, which uses material cross sections group-collapsed from the C5G7 benchmark. As in the monoenergetic case, the predictions of the Fourier analysis agree with direct simulations (though direct MC-CMFD results are considerably noisier for the two-group case, and thus cannot be used to draw definitive conclusions). To investigate how accurately the two-group model predicts the convergence rate of more realistic energy group structures, we use surrogate $\mathrm{S}_{N}$-CMFD simulations to calculate numerical spectral radius estimates for a case with seven-group MC accelerated by two-group CMFD (which we call the $7 \mathrm{~g} / 2 \mathrm{~g}$ iteration scheme). Results show that the $7 \mathrm{~g} / 2 \mathrm{~g}$ iteration remains stable over a larger range of coarse grid sizes than the two-group Fourier analysis predicts; thus, few-group Fourier analyses should not be used to quantitatively predict the spectral radius of many-group simulations.

The Fourier analysis and direct numerical simulations show that the spectral radius (i) increases monotonically as the coarse mesh optical thickness increases, and (ii) decreases monotonically as the scattering ratio increases (in the monoenergetic case). Our results also show that the non-random MC-CMFD $k$-eigenvalue iteration becomes unstable when the coarse mesh exceeds a critical value (which, for the monoenergetic problem considered in this paper, depends on the scattering ratio $c$ ). These results imply that the MC-CMFD method should not be used for problems in which the non-random method is unstable. Fortunately, our results show that if the non-random MC-CMFD method is unstable, it can be stabilized by using a finer coarse grid. (Of course, this increases the cost of the low-order CMFD calculation.) Previous work [5] has shown that it is also possible to stabilize the low-order calculation by accumulating MC tallies across several cycles; however, this reintroduces some degree of inter-cycle correlation, and can bias the solution if CMFD feedback is continued during active cycles.

The analysis in this paper does not account for continuous-energy crosssections, stochastic error or multidimensional transport. Thus, we cannot predict the effect of stochastic errors on convergence, nor can we quantitatively predict spectral radii for continuous energy or multidimensional problems. It is not clear whether our theory can be generalized to include stochastic errors. Conceptually, it is possible to extend the Fourier analysis to more complicated problems. However, the algebraic difficulties with even the simple 1-D, monoenergetic and two-group problems treated here are significant; extending this analysis to more complicated problems would be difficult. Fortunately, the work in this paper points to an alternate and more practical method for calculating spectral radii for realistic (multidimensional, heterogeneous) problems: use a surrogate $\mathrm{S}_{\mathrm{N}^{-}}$CMFD code. Specifically, implement the non-random MCCMFD algorithm in an $\mathrm{S}_{\mathrm{N}}$ code and use the spectral radius estimates obtained from that. The 1-D results in this paper show excellent agreement between the Fourier analysis and the $\mathrm{S}_{\mathrm{N}}$-CMFD surrogate code, and there is no reason to think that this would not be true for more complicated problems. However, this task cannot be considered here.

In conclusion, we hope that the results derived in this paper will provide a 
more rigorous theoretical basis for applications of the MC-CMFD method. In particular, we hope that users of the method will be more aware of (previously unrecognized) stability issues, and that adequate steps will be taken to avoid simulations in which the non-random MC-CMFD method has a spectral radius which is greater than, or too close to, unity.

\section{ACKNOWLEDGMENT}

We gratefully acknowledge support from the Consortium for Advanced Simulation of Light Water Reactors (www.casl.gov), an Energy Innovation Hub (http://www.energy.gov/hubs) for Modeling and Simulation of Nuclear Reactors under U.S. Department of Energy Contract No. DE-AC05-00OR22725.

\section{REFERENCES}

[1] M.J. Lee, H.G. Joo, D. Lee, and K. Smith, "A Feasibility Study of CMFD Acceleration in Monte Carlo Eigenvalue Calculation," Trans. Kor. Nucl. Soc. Autumn Meeting, Gyeongju, Korea, October 29-30, 2009 (2009).

[2] M.T.H. Young, F.B. Brown, B.C. Kiedrowski, and W.R. Martin, "Coarse Mesh Finite Difference in MCNP5," Los Alamos National Laboratory, LA-UR-11-04384 (2011).

[3] M.J. Lee, H.G. Joo, D. Lee, and K. Smith, "Investigation of CMFD Accelerated Monte Carlo Eigenvalue Calculation with Simplified Low Dimensional Multigroup Formulation," Proc. PHYSOR 2010, Pittsburgh, Pennsylvania, May 9-14, 2010 (2010).

[4] M.J. Lee, H.G. Joo, D. Lee, and K. Smith, "Monte Carlo Reactor Calculation with Substantially Reduced Number of Cycles," Proc. PHYSOR 2012, Knoxville, Tennessee, April 15-20, 2012 (2012).

[5] M.J. Lee, H.G. Joo, D. Lee, and K. Smith, "Coarse Mesh Finite Difference Formulation for Accelerated Monte Carlo Eigenvalue Calculation," Ann. Nucl. Energy, 65, 101 (2014).

[6] P.K. Romano, et al., "OpenMC: A State-of-the-Art Monte Carlo Code for Research and Development," Ann. Nucl. Energy, (2014).

[7] M.L. Adams and E.W. Larsen, "Fast Iterative Methods for DiscreteOrdinates Particle Transport Calculations," Prog. Nucl. Energy, 40, 3 (2002).

[8] E.W. Larsen and B.W. Kelley, "The Relationship Between the CoarseMesh Finite Difference and the Coarse-Mesh Diffusion Synthetic Acceleration Methods," Nucl. Sci. Eng., 178, 1 (2014). 
[9] K.S. Smith and J.D. Rhodes, "Full-Core, 2-D, LWR Core Calculations with CASMO-4E," Proc. PHYSOR 2002, Seoul, Korea, October 7-10, 2002 (2002).

[10] D. Lee, T.J. Downar, and Y. Kim, "Convergence Analysis of the Nonlinear Coarse-Mesh Finite Difference Method for One-Dimensional Fixed-Source Neutron Diffusion Problem," Nucl. Sci. Eng., 147, 127 (2004).

[11] S.G. Hong, K.-S. Kim, and J.S. Song, "Fourier Convergence Analysis of the Rebalance Methods for Discrete Ordinates Transport Equations in Eigenvalue Problems," Nucl. Sci. Eng., 164, 33 (2010).

[12] K. Keady, "Stabilization and Acceleration Methods for Monte Carlo Reactor Core $k$-Eigenvalue Problems," Ph.D. Dissertation (2016).

[13] "Benchmark on Deterministic Transport Calculations without Spatial Homogenisation", Organization for Economic Cooperation and Development/Nuclear Energy Agency, ISBN 92-64-02139-6, (2003).

[14] B. R. Herman, B. Forget, and K. Smith, "Progress toward Monte Carlothermal hydraulic coupling using low-order nonlinear diffusion acceleration methods.," Ann. Nucl. Energy (2014). 